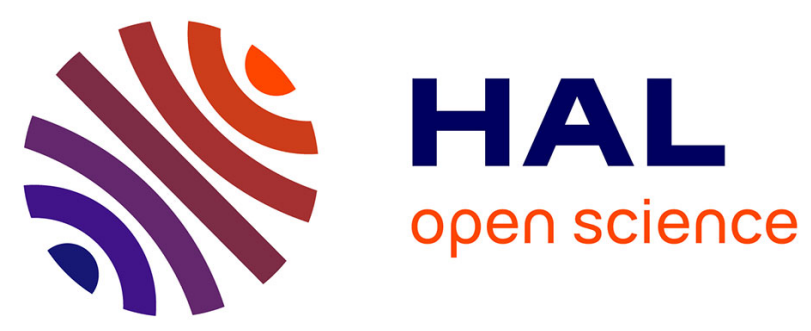

\title{
Multimodal Nonlinear Optical Imaging of MoS2 and MoS2-Based van der Waals Heterostructures.
}

Dawei Li, Wei Xiong, Lijia Jiang, Zhiyong Xiao, Hossein Rabiee Golgir, Mengmeng Wang, Xi Huang, Yunshen Zhou, Zhe Lin, Jingfeng Song, et al.

\section{- To cite this version:}

Dawei Li, Wei Xiong, Lijia Jiang, Zhiyong Xiao, Hossein Rabiee Golgir, et al.. Multimodal Nonlinear Optical Imaging of MoS2 and MoS2-Based van der Waals Heterostructures.. ACS Nano, 2016, 10 (3), pp.3766-3775. 10.1021/acsnano.6b00371 . hal-01299527

\section{HAL Id: hal-01299527 \\ https://hal.science/hal-01299527}

Submitted on 10 Mar 2021

HAL is a multi-disciplinary open access archive for the deposit and dissemination of scientific research documents, whether they are published or not. The documents may come from teaching and research institutions in France or abroad, or from public or private research centers.
L'archive ouverte pluridisciplinaire $\mathbf{H A L}$, est destinée au dépôt et à la diffusion de documents scientifiques de niveau recherche, publiés ou non, émanant des établissements d'enseignement et de recherche français ou étrangers, des laboratoires publics ou privés. 


\section{Multimodal Nonlinear Optical Imaging of $\mathrm{MoS}_{2}$}

\section{and $\mathrm{MoS}_{2}$-Based van der Waals}

\section{Heterostructures}

Dawei Li ${ }^{\dagger},{ }^{ \pm}$Wei Xiong, ${ }^{\dagger},{ }^{\ddagger}{ }^{ \pm}$Lijia Jiang, ${ }^{\dagger}$ Zhiyong Xiao, ${ }^{\dagger}$ Hossein Rabiee Golgir,${ }^{\dagger}$ Mengmeng Wang, ${ }^{\dagger}$ Xi Huang, ${ }^{\dagger}$ Yunshen Zhou, ${ }^{\dagger}$ Zhe Lin, ${ }^{\dagger}$ Jingfeng Song, ${ }^{\ddagger}$ Stephen Ducharme, ${ }^{\ddagger}$ Lan Jiang, ${ }^{\neq}$Jean-Francois Silvain, ${ }^{*}$ and Yongfeng $L u^{\dagger}, *$

${ }^{\dagger}$ Department of Electrical and Computer Engineering, University of Nebraska-Lincoln, Lincoln, NE 68588-0511, United States

$\S$ Wuhan National Laboratory for Optoelectronics, Huazhong University of Science and Technology, 1037 Luoyu Road, Wuhan 430074, China

Department of Physics and Astronomy, University of Nebraska-Lincoln, Lincoln, NE 68588-0511, United States

${ }^{*}$ School of Mechanical Engineering, Beijing Institute of Technology, Beijing, 100081, China

\# Institut de Chimie de la Matière Condensée de Bordeaux, Avenue du Docteur Albert Schweitzer F-33608 Pessac Cedex, France

${ }^{ \pm}$D.W. Li and W. Xiong contributed equally to this work.

* Address correspondence to: ylu2@unl.edu

KEYWORDS: Nonlinear optical imaging, transition metal dichalcogenides, van der Waals heterostructure, four-wave mixing, sum-frequency generation, second-harmonic generation, interlayer coupling 
ABSTRACT: Van der Waals layered structures, notably the transitional metal dichalcogenides (TMDs) and TMD-based heterostructures, have recently attracted immense interest due to their unique physical properties and potential applications in electronics, optoelectronics, and energy harvesting. Despite the recent progress, it is still a challenge to perform comprehensive characterizations of critical properties of these layered structures, including crystal structures, chemical dynamics, and interlayer coupling, using a single characterization platform. In this study, we successfully developed a multimodal nonlinear optical imaging method to characterize these critical properties of molybdenum disulfide $\left(\mathrm{MoS}_{2}\right)$ and $\mathrm{MoS}_{2}$-based heterostructures. Our results demonstrate that $\mathrm{MoS}_{2}$ layers exhibit strong four-wave mixing (FWM), sum-frequency generation (SFG), and second-harmonic generation (SHG) nonlinear optical characteristics. We believe this is the first observation of FWM and SFG from TMD layers. All three kinds of optical nonlinearities are sensitive to layer numbers, crystal orientation, and interlayer coupling. The combined and simultaneous SHG/SFG-FWM imaging is not only capable of rapid evaluation of crystal quality and precise determination of odd-even layers but also provides in situ monitoring of the chemical dynamics of thermal oxidation in $\mathrm{MoS}_{2}$ and interlayer coupling in $\mathrm{MoS}_{2}$-graphene heterostructures. This method has the advantages of versatility, high fidelity, easy operation, and fast imaging, enabling comprehensive characterization of van der Waals layered structures for fundamental research and practical applications. 
Van der Waals layered structures, in particular the two-dimensional (2D) transition metal dichalcogenide (TMD) semiconductors (such as $\mathrm{MoS}_{2}$ ), have recently attracted significant research and industrial interest due to their outstanding electronic and optical properties and their potential applications for the next-generation of flexible field-effect transistors (FET), ${ }^{1-5}$ logic circuits, ${ }^{6,7}$ photodetector and photovoltaic devices, ${ }^{8-11}$ solar cells, ${ }^{12}$ catalysis,${ }^{13,14}$ energy storage, ${ }^{15,16}$ sensors, ${ }^{17-19}$ spintronics, ${ }^{20-22}$ and memory ${ }^{23-25}$ devices. While pure 2D TMD semiconductors have been gaining more attention, there have also been advances in designing heterostructures with various functionalities which use other 2D materials, such as graphene, in conjunction with TMDs in pursuit of novel devices utilizing the unique properties of more than one material. ${ }^{26}$ For example, FETs composed of a TMD-graphene heterostructure can combine the advantages of high carrier mobility in graphene with the permanent bandgaps of TMDs. ${ }^{27} \mathrm{MoS}_{2}$-graphene heterostructures can be used as nonvolatile memory cells by combining the unique semiconducting properties of monolayer $\mathrm{MoS}_{2}$ with the high conductivity of graphene. ${ }^{24}$ In addition, it has been recently reported that TMD-graphene heterostructures may create barrier-free contacts to TMD materials. ${ }^{28,29}$ Overall, the diverse performances of these TMD and TMD-graphene heterostructures are significantly determined by their layer thickness, crystalline symmetry, interface quality, interlayer coupling, and crystalline quality. Therefore, fast, scalable, and nondestructive methods for comprehensive characterizations are in high demand.

Despite the recent progress, it is still a challenge for comprehensive characterizations of these 2D TMDs using a single characterization method. Existing methods, such as atomic force microscopy (AFM), transmission electron microscopy (TEM), optical microscopy (OM), and Raman and photoluminescence (PL) spectroscopy, have been widely used as characterization tools. Compared to time-consuming and complicated AFM and TEM analyses, most of the optical spectroscopic methods allow for rapid, noninvasive, and quantitative characterizations 
of 2D TMDs. Raman spectroscopy is commonly used to accurately identify the layer thickness as well as to study the crystal quality of 2D TMDs. Taking $\mathrm{MoS}_{2}$ as an example, $\mathrm{MoS}_{2}$ has two main Raman active modes, out-of-plane $A_{1 g}$ and in-plane $E_{2 g}^{1}$ vibrational modes. The frequency or frequency-shift difference between both modes can be used as a film thickness indicator. ${ }^{30,31}$ The low-frequency interlayer breathing $\left(B_{2 g}^{2}\right)$ and shear $\left(E_{2 g}^{2}\right)$ modes can also be used to examine the structural properties of few-layer $\mathrm{MoS}_{2}$ films and determine their layer thickness. ${ }^{32}$ PL spectroscopy is also frequently used in characterization of 2D TMDs to determine their layer thickness, quality, and crystallinity based on peak position or intensity of PL peaks. ${ }^{33,34}$ However, both Raman and PL spectroscopy are sensitive to temperature, defects, doping, and substrate materials. Since the contrast is relatively low, it is impossible to accurately identify numbers of atomic layers, especially for thick 2D TMDs. In addition, Raman, PL, and AFM are not straightforward, effective methods for determining whether TMDs contain 2D crystalline structures of large grain sizes.

Recently, nonlinear optical responses, notably second-harmonic generation (SHG), in 2D TMDs have gained great interest. ${ }^{35-39}$ SHG has become a robust characterization tool for determining structural symmetry, crystal orientation, and layer stacking of monolayer to fewlayer TMDs. The SHG intensity from 2D TMDs displays an even-odd oscillation decay with increasing layer thickness, where the intense SHG is only observed on odd-numbered layers. Therefore, SHG cannot be used as an accurate film thickness and parity indicator. So far, other higher-order optical nonlinearities of TMDs, such as four-wave mixing (FWM) and sum-frequency generation (SFG), and their applications in optical characterizations have been rarely studied. The combination of different types of nonlinear optical effects is expected to offer a comprehensive approach to fundamental research and practical applications of 2D TMDs. 
As the structural and electronic properties of the family of group-VI 2D TMDs (such as $\mathrm{MoS}_{2}, \mathrm{WSe}_{2}, \mathrm{WS}_{2}$, and $\mathrm{MoSe}_{2}$ ) are similar, $\mathrm{MoS}_{2}$ was chosen as a model case in this study. We investigated the nonlinear optical properties of $\mathrm{MoS}_{2}$ and $\mathrm{MoS}_{2}$-based heterostructures using multiphoton nonlinear optical microscopy, where the second- (SHG, SFG) and thirdorder (FWM) optical nonlinearities were simultaneously observed (Figure 1a). This was also the first observation of FWM and SFG from $\mathrm{MoS}_{2}$ atomic thin layers. All three types of the nonlinearities are sensitive to the number of atomic layers, crystal orientation, crystal quality, and interlayer coupling, while SHG and SFG are sensitive to films with odd-numbered layers but not to those with even-numbered layers. Therefore, the combined SHG/SFG-FWM imaging not only allows for rapid evaluation of crystal quality and precise determination of odd-even layers but also for the in situ monitoring of chemical reactions in $\mathrm{MoS}_{2}$, such as thermal oxidation. The nonlinear optical properties of $\mathrm{MoS}_{2}$-graphene heterostructures were also demonstrated. Interestingly, $\mathrm{MoS}_{2}$-graphene heterostructures showed strong quenching of SHG-SFG signals from $\mathrm{MoS}_{2}$, indicating strong interlayer coupling between $\mathrm{MoS}_{2}$ and graphene. This fast and noninvasive multimodal nonlinear optical imaging approach is applicable to a broad range of 2D TMDs and TMD-based heterostructures to elucidate their fundamental structural and interlayer coupling properties.

\section{RESULTS AND DISCUSSION}

\section{Multimodal nonlinear spectroscopic imaging of $\mathrm{MoS}_{2}$ atomic layers}

Few-layer $\mathrm{MoS}_{2}$ flakes were exfoliated from bulk $\mathrm{MoS}_{2}$ crystals. The number of atomic layers was determined using optical microscopy and Raman spectroscopy (inset in Figure 1a). By examining the frequency difference between $E_{2 g}^{1}\left(\sim 383.8 \mathrm{~cm}^{-1}\right)$ and $A_{1 g}$ modes $(\sim 407.1$ $\mathrm{cm}^{-1}$ ), a third layer in a $\mathrm{MoS}_{2}$ sheet was identified. ${ }^{31}$ The homemade pump-probe microscopic system was employed to detect the nonlinear optical properties (Supporting Information, Figure S1). The pump-probe laser beams were linearly polarized, collinear, and focused onto 
the $\mathrm{MoS}_{2}$ samples. Figure $1 \mathrm{~b}$ shows the multimodal nonlinear optical spectrum obtained from the trilayer $\mathrm{MoS}_{2}$ thin film (black curve) by introducing pump-probe laser beams with wavelengths of $\lambda_{\text {pump }}(800 \mathrm{~nm})$ and $\lambda_{\text {probe }}(820$ to $1100 \mathrm{~nm})$ (Supporting Information, Figure S2), respectively. The resulting spectrum consisted of three signal peaks: a sharp peak at a wavelength of $\sim 400 \mathrm{~nm}$ with $\Delta \lambda=2 \mathrm{~nm}$, a strong peak at a wavelength of $\sim 450 \mathrm{~nm}$ with $\Delta \lambda$ $=12 \mathrm{~nm}$, and a broad peak at a wavelength of $\sim 655 \mathrm{~nm}$ with $\Delta \lambda=19 \mathrm{~nm}$, labeled as "Peak A", Peak B" and "Peak C", respectively. By turning the probe beam off, only "Peak A" around $400 \mathrm{~nm}$ was observed (red curve), while only a weak and broad peak lower than 450 nm (not "Peak B") was detected after turning the pump beam off (blue curve). The results described above indicate that "Peak A" was only associated with the pump beam, while "Peak B" and "Peak C" were related to both the pump and probe beams. Further analysis showed that the "Peak A" signal was always detected at double the pump beam frequency (Figure 1c). It also followed a square power law behavior as a function of pump laser power (Supporting Information, Figure S3b), which confirmed that "Peak A" was the secondharmonic emission: $2 \omega_{\text {pump }}$.

In our system, only part of the probe pulse temporally overlaps with the pump pulse because of the complex temporal structure of the continuum probe pulses (Supporting Information, Figure S2b). Thus to verify the origination of the "Peak B" and "Peak C" signals, we measured wavelength-dependent emission spectra by adjusting the pump-probe delays. Figures 1d and 1e show the measured "Peak B" and "Peak C" nonlinear optical spectra from the trilayer $\mathrm{MoS}_{2}$ as functions of emission wavelength $\lambda_{e}$ for several combinations of the pump-probe wavelengths. All of the emission spectra were obtained by adjusting the delay time for each pump wavelength (Supporting Information, Figure S3). In all of the measurements (Figures 1d and 1e), a clear resonant emission peak was observed at the 
wavelength corresponding to the frequency $\omega_{e}=\omega_{\text {pump }}+\omega_{\text {probe }}$ for "Peak B" (Figure 1d) and $\omega_{e}=2 \omega_{\text {pump }}-\omega_{\text {probe }}$ for "Peak C" (Figure 1e), indicating that the "Peak B" and "Peak C" signals were generated due to the second- (SFG) and third-order nonlinear optical FWM processes, respectively. Therefore, $\mathrm{MoS}_{2}$ can be used as frequency converters to create three different waves at the same time from two incident frequencies of $\omega_{\text {pump }}$ and $\omega_{\text {probe }}$ : $2 \omega_{\text {pump }}(\mathrm{SHG}), \omega_{\text {pump }}+\omega_{\text {probe }}(\mathrm{SFG})$, and $2 \omega_{\text {pump }}-\omega_{\text {probe }}(\mathrm{FWM})$. The investigation of polarization dependence of nonlinear optical signals from $\mathrm{MoS}_{2}$ also confirmed the secondorder nonlinear optical natures of SHG and SFG, and the third-order nonlinear optical nature of FWM (see Supporting Information, Figures S4 and S5). Although we focused our research on the nonlinear optical properties of $\mathrm{MoS}_{2}$ atomic layers, our results are generically applicable to a large class of 2D TMDs (e.g., we also observed SHG-SFG-FWM signals in $\mathrm{MoSe}_{2}$ atomic flakes, see Supporting Information, Figure S6).

The results described above show the feasibility of simultaneous measurement of SHG, SFG, and FWM signals from a single spot of $\mathrm{MoS}_{2}$ flakes. Few-layer $\mathrm{MoS}_{2}$ flakes with different thicknesses were exfoliated from bulk $\mathrm{MoS}_{2}$ crystals. The number of atomic layers was first verified using OM, AFM, Raman, and PL spectroscopy (see Supporting Information, Figure S7). A MoS 2 flake consisted of mono (1L) to six (6L), eight (8L), and multi-layers (ML); and their respective layer numbers are shown in Figure 2. Nonlinear optical microscopy was then employed to conduct simultaneous FWM-SHG-SFG multimodal optical imaging, which showed the different thickness-dependent optical contrasts, as marked in the corresponding figures (Figures $2 \mathrm{a}-2 \mathrm{c}$ ). Figures $2 \mathrm{a}$ and $2 \mathrm{~d}$ show the FWM imaging and the dependence of the FWM intensity on the $\mathrm{MoS}_{2}$ film thickness. It was found that the FWM intensity was proportional to the number of $\mathrm{MoS}_{2}$ layers over a wide range of thicknesses, extending to $\sim 13$ atomic layers. When the thickness was beyond $\sim 13 \mathrm{~L}$, the FWM intensity 
started to decay exponentially as the $\mathrm{MoS}_{2}$ layer thickness increased (Supporting Information, Figure S8). The linear intensity increase observed $(\leq 13 \mathrm{~L})$ implies that the total FWM intensity was the sum of contributions from individual atomic layers. The FWM contrast was sensitive to thickness changes of a single layer. Therefore, FWM can provide a simple way to measure thickness variations in few-layer $\mathrm{MoS}_{2}$. The exponential intensity decrease for thick flakes $(>13 \mathrm{~L})$ can be explained as follows. First, the reflection of the incident pump-probe beams on the sample surface reduces the probability of transformation of the pump-probe lights into nonlinear optical signals. Second, the nonlinear optical signal generated inside a thick flake can also be reabsorbed. ${ }^{40}$

Similar to the previous report, the SHG of odd-layered $\mathrm{MoS}_{2}$ showed an exponential decrease with an increase in the layer numbers, while the SHG of even-numbered layers vanished due to the restoration of inversion symmetry (Figures $2 \mathrm{~b}$ and $2 \mathrm{e}$ ). ${ }^{37,41}$ As seen in Figures $2 \mathrm{~b}$ and $2 \mathrm{c}$, the contrasts of both the SFG and SHG images from $\mathrm{MoS}_{2}$ are largely similar, as both modalities are sensitive to the elements of second-order nonlinearity $\chi^{(2)}$. Features seen in the SHG images are reproduced in the SFG images. Nonetheless, some differences can be observed as well. Figure $2 \mathrm{f}$ shows the SFG intensity dependence on $\mathrm{MoS}_{2}$ layer numbers. The SFG intensity of odd-numbered layers increased slightly when the thickness increased from monolayer to trilayer and then rapidly decreased with further increases in the layer numbers, different from the SHG intensity observed from $\mathrm{MoS}_{2}$ layers. It was confirmed, by numerous tests on different samples, that the maximum SFG intensity always appear from the trilayer $\mathrm{MoS}_{2}$ (Figures 2c, Supporting Information, Figure S9). However, the basic mechanism behind this phenomenon is still not clear at this point and will require additional study. Based on the analyses described above, compared to the pure SHG and SFG which are only sensitive to the odd-layered TMDs, the combined second-(SHG or SFG) and third-order (FWM) optical responses allowed us to more precisely identify the 
layer numbers and measure their evolution, which was also more accurate and much faster than Raman and PL spectroscopy.

In addition to layer numbers, we also investigated the combined and simultaneous multimodal nonlinear optical imaging for 1) $\mathrm{MoS}_{2}$ crystal quality evaluation and 2) in situ monitoring of chemical reactions in $\mathrm{MoS}_{2}$.

\section{SHG-FWM imaging for crystal quality evaluation of $\mathrm{MoS}_{2}$ films grown by direct} sulfurization

It is important to consider the possible defects, degree of crystallinity, and their effects on material properties for $\mathrm{MoS}_{2}$ thin films grown using the solid-state sulfurization (SSS) method. ${ }^{42,} 43$ To explore the potential of SHG-FWM imaging for rapid and quantitative quality evaluation, we synthesized $\mathrm{MoS}_{2}$ thin films at different temperatures. Details of the film growth are provided in Supporting Information, Figure S10.

Raman and PL characterizations (Supporting Information, Figure S11) were used to qualitatively demonstrate the successful growth of $\mathrm{MoS}_{2}$ with improved structural quality from relatively low $\left(800^{\circ} \mathrm{C}\right)$ to sufficiently high $\left(1100^{\circ} \mathrm{C}\right)$ temperatures. The same samples were then examined using the simultaneous SHG-FWM imaging (Figure 3, Supporting Information, Figure S12). There were no SHG and FWM signals at a low temperature (800 $\left.{ }^{\circ} \mathrm{C}\right)$. At an intermediate temperature $\left(950{ }^{\circ} \mathrm{C}\right)$, very weak $\mathrm{SHG}$ and $\mathrm{FWM}$ signals were observed (Supporting Information, Figure S12). At a high temperature $\left(1100{ }^{\circ} \mathrm{C}\right)$, some strong SHG and FWM spots with different intensities were observed and randomly distributed on the film surfaces, as shown in Figures $3 \mathrm{a}$ and 3b. AFM characterization revealed a $\mathrm{MoS}_{2}$ film consisting of quasi-continuous particles at $800-950{ }^{\circ} \mathrm{C}$ (Supporting Information, Figure S12) and a hole-structured film formation at $1100{ }^{\circ} \mathrm{C}$ (Figure 3d). Closer observation revealed that the layered $\mathrm{MoS}_{2}$ nanoflakes [with a lateral size of approximately 
several hundred nanometers and typical layer numbers from 1 to $\sim 4 \mathrm{~L}$ (Supporting Information, Figure S13)] were formed in these holes, which was reflected by the SHG-FWM imaging. Both SHG and FWM were very sensitive to the layered crystalline structures but not to the noncrystalline structures. Figure 3e shows the SHG-FWM images of two typical crystal spots: A and B. The parallel polarized SHG intensity at these two spots as functions of the polarization angle of the pump beam are shown in Figure $3 \mathrm{f}$. A clear four-fold symmetry with different SHG peak positions was observed, confirming that both A and B spots were crystallized but with a crystal orientation difference of $\sim 35^{\circ}$.

To provide a more quantitative analysis of the SSS-grown $\mathrm{MoS}_{2}$ grown at $1100{ }^{\circ} \mathrm{C}$, superposed nonlinear optical imaging was performed. As we know, the FWM is sensitive to all layered crystal structures. FWM intensity differences among $\mathrm{MoS}_{2}$ nanoflakes reflected their size dispersion and layer numbers (Figure 3a). Strong SHG signals only revealed oddnumbered layers but not even-numbered layers (Figure 3b). It is exciting that the superposed FWM-SHG imaging allowed us to simultaneously identify both even and odd layers with a depth resolution of a single atomic layer (Figure 3c). By analyzing the superposed FWMSHG, we can even determine the specific number of layers for each nanoflake, according to the linear (exponential decrease) relationship between the FWM (SHG) intensity and $\mathrm{MoS}_{2}$ layer thickness, as described above. In this study, eleven nanoflakes were randomly selected (see Figure 3c) and sorted from thick to thin (corresponding FWM signal from strong to weak) using Arabic numbers (Figure 3g). Nanoflake No. 11 with the strongest (weakest) SHG (FWM) signal must be a monolayer based on the AFM analysis described above. Therefore, the thickness (or layer numbers) of other nanoflakes was successively determined, as shown in Figure 3g. Thus, we can directly visualize the odd-even and number of layers, which provides key information for optimizing synthesis strategies for growing large-area, uniform $\mathrm{MoS}_{2}$ layers. 
Nonlinear optical mapping to study chemical dynamics of thermal oxidation in few-layer $\mathrm{MoS}_{2}$

Investigation of the oxidation process in TMDs is very important due to its great impact on the performance of TMD-based devices, ${ }^{44}$ which has been extensively studied during the last two years. ${ }^{45-47}$ Here, the combined SHG-FWM was used for structural and electronic imaging to monitor the oxidation dynamics of $\mathrm{MoS}_{2}$ and its temporal and spatial evolution.

To understand the oxidation dynamics, we repeatedly oxidized $\mathrm{MoS}_{2}$ samples at a temperature of $\sim 310{ }^{\circ} \mathrm{C}$ for a number of steps in a furnace with an annealing time of $t_{a}$ in air. SHG-FWM images were acquired in between these steps. Figure 4a displays simultaneously acquired SHG-FWM images of a bilayer $\mathrm{MoS}_{2}$ sample during the thermal oxidation process. Before the annealing, negligible SHG response and strong FWM intensity were observed, further confirming a pure $\mathrm{MoS}_{2}$ component with a bilayer structure. After a heating time $t_{a}$ of $\sim 0.5 \mathrm{~h}$, the SHG signal at the edges increased $\sim 300 \%$ (Figure $4 \mathrm{~b}$ ), while no obvious change was observed for the optical and FWM imaging. The change in the SHG clearly marks the phase transition from $\mathrm{MoS}_{2}$ to $\mathrm{MoO}_{3}$, which can be expressed as: $2 \mathrm{MoS}_{2}+7 \mathrm{O}_{2} \rightarrow 2 \mathrm{MoO}_{3}+$ $4 \mathrm{SO}_{2}{ }^{44}$ After a heating time of $\sim 1.5 \mathrm{~h}$, strong SHG signals randomly appeared around the edges and in the interior of the sample, while the corresponding FWM signals were reduced to half of the initial intensity, indicating that the oxidation resulted in a decrease in thickness from bilayer to monolayer. The spots with strong local SHG in the center area suggest that the oxidation took place at the top surface and started from lattice defects. For the next $\sim 2.5$ h of oxidation, both SHG and FWM signals at the edge started to disappear, indicative of full oxidation and decomposition of the monolayer $\mathrm{MoS}_{2}$. Due to the stability of the center area, the SHG signal continuously increased and the FWM signal changed little even with an annealing time of more than $3 \mathrm{~h}$ (Figure $4 \mathrm{~b}$ ). This indicates the central area of the sample 
gradually became a complete $\mathrm{MoS}_{2}$ monolayer, as further confirmed by Raman spectroscopy (Figure 4c).

We have also examined the role of layer numbers in the thermal oxidation dynamics (Supporting Information, Figure S14). It was found that the oxidation (removal) rates in the monolayer, bilayer, and trilayer surfaces were almost the same. Based on the analyses above, we expect that combined multimodal nonlinear optical imaging holds promise as a noninvasive tool for monitoring chemical reactions in 2D materials.

\section{Nonlinear optical imaging of interlayer coupling in $\mathrm{MoS}_{2}$-graphene heterostructures}

$\mathrm{MoS}_{2}$-graphene as a typical TMD-based heterostructure has attracted great interest as it offers excellent electronic and optical properties. Nevertheless, its nonlinear optical properties have not been studied until now. Figures $5 \mathrm{a}$ and $5 \mathrm{~b}$ show the optical and AFM images of a $\mathrm{MoS}_{2}$-graphene heterostructure. The thicknesses of the $\mathrm{MoS}_{2}$ and graphene were measured to be a monolayer and a bilayer, respectively. The SHG, SFG, and FWM images of the same sample are shown in Figure 5c-5e, where the individual monolayer $\mathrm{MoS}_{2}$, bilayer graphene, and the stacking regions are marked with dotted lines. The SHG-SFG intensity from $\mathrm{MoS}_{2}$ flake was strong and uniform, while a decreased SHG-SFG intensity was observed in the stacking region (Figures $5 \mathrm{c}$ and $5 \mathrm{~d}$ ), which was only about half that of the monolayer $\mathrm{MoS}_{2}$ alone (Figures 5f and 5g). In addition, the weak "SHG-SFG" signal observed in the graphene region was actually a part of the nonlinear broadband photoluminescence of the graphene induced by the pump-probe fs laser irradiation (Supporting Information, Figure S15), which arose from the radiative recombination of broad distribution of nonequilibrium electrons and holes, generated by rapid scattering between photo-excited carriers within tens of femtoseconds after the optical excitation. ${ }^{48}$ As shown in Figures $5 \mathrm{e}$ and $5 \mathrm{~h}$, the FWM intensity of the monolayer $\mathrm{MoS}_{2}$ was much weaker than that of 
the graphene due to the negligible third-order nonlinear susceptibility, $\chi^{(3)}$, compared to that of graphene. ${ }^{49}$ Therefore, the FWM intensity from the stacking region was similar to that of individual graphene. The results described above were also found in more $\mathrm{MoS}_{2}$-graphene heterostructures (Supporting Information, Figure S16).

It is known that SHG intensity strongly depends on the stacking angle (relative crystal orientation between adjacent layers) for heterostructures made of noncentrosysmmetric layered materials, such as $\mathrm{MoS}_{2}-\mathrm{MoS}_{2}$ and $\mathrm{WSe}_{2}-\mathrm{MoS}_{2} \cdot{ }^{50}$ However, this law does not apply to the $\mathrm{SHG} / \mathrm{SFG}$ responses in the $\mathrm{MoS}_{2}$-graphene heterostructure because graphene is a centrosymmetric material. This was further verified by the polarization-resolved SHG, as shown in Figure 5i, where a clear, coherent, and fourfold symmetry was observed at the monolayer $\mathrm{MoS}_{2}$ and the stacking regions, suggesting that two different regions were crystallized in the same crystalline orientation.

The strongly suppressed SHG-SFG from the $\mathrm{MoS}_{2}$-graphene heterostructure was presumably due to the strong interlayer coupling between the $\mathrm{MoS}_{2}$ and graphene layers. Calculation indicated that the SHG (SFG) signal in $\mathrm{MoS}_{2}$ highly depended on the electronhole interaction..$^{51}$ Due to the strong interlayer coupling, ultrafast photo-excited electrons and holes from the $\mathrm{MoS}_{2}$ layer efficiently transferred to the graphene layer during the SHG/SFG process. They combined through the rapid nonradiative recombination near the Dirac point of graphene, which led to a much reduced SHG/SFG emission from $\mathrm{MoS}_{2}$. We observed a significant Raman mode $A_{1 g}$ blue shift (by $\sim 1.4 \mathrm{~cm}^{-1}$ ) and an obvious PL peak intensity decrease (by $\sim 20 \%$ ) in the stacking region compared with those in the monolayer $\mathrm{MoS}_{2}$ (Supporting Information, Figure S17), which indicates the possible interlayer charge transfer between $\mathrm{MoS}_{2}$ and graphene. ${ }^{27,52}$ However, the strain, temperature, substrate materials, and capping environment also have significant effects on the Raman and PL signals of monolayer 
$\mathrm{MoS}_{2}$. Thus, electric and photocurrent measurements were conducted to further prove the interlayer coupling effects between the $\mathrm{MoS}_{2}$ and graphene layers. ${ }^{53,54} \mathrm{Ti} / \mathrm{Au}(5 / 50 \mathrm{~nm})$ thin film was deposited on each layer by standard electron-beam evaporation (inset in Figure 5j). Figure $5 \mathrm{j}$ presents the current versus drain voltage of the $\mathrm{MoS}_{2}-$ graphene heterostructure in the dark at $\mathrm{V}_{\mathrm{G}}=0 \mathrm{~V}$. The $\mathrm{MoS}_{2}$-graphene heterostructure exhibited diode-rectifying behavior benefited from the interlayer coupling and charge transfer process, which was consistent with the previous report. ${ }^{53}$ Photocurrent line mapping was also conducted on the $\mathrm{MoS}_{2}-$ graphene heterostructure $\left(\mathrm{V}_{\mathrm{SD}}=-0.2 \mathrm{~V}\right)$, which was recorded by scanning a focused $405 \mathrm{~nm}$ laser beam with a spot size and power of $\sim 1 \mu \mathrm{m}$ and $\sim 5 \mu \mathrm{W}$ (inset in Figure $5 \mathrm{j}$ ). ${ }^{55}$ The strongest photocurrent response was observed in the stacking region (Figure 5k), indicating that spontaneous charge separation occurred at the interface of $\mathrm{MoS}_{2}$ and graphene. ${ }^{54}$ The electric and scanning photocurrent measurements therefore further confirmed a strong coupling between the monolayer $\mathrm{MoS}_{2}$ and bilayer graphene.

It has been reported that the interlayer coupling greatly depends on the layer distance. ${ }^{56,57}$ We, therefore, designed a sandwich structure by inserting an isolating $\mathrm{Al}_{2} \mathrm{O}_{3}$ thin film of $\sim 1$ $\mathrm{nm}$ thickness to investigate the effect of the layer distance on the nonlinear optical properties of the $\mathrm{MoS}_{2}$-graphene heterostructure (see Supporting Information, Figure S18). The interlayer coupling was almost negligible for the $\mathrm{MoS}_{2}$-graphene heterostructure with the $\sim 1$ $\mathrm{nm} \mathrm{Al}_{2} \mathrm{O}_{3}$ insertion, as indicated by the SHG and SFG intensities (Supporting Information, Figure S18f), which were nearly the same between $\mathrm{MoS}_{2}$ alone and $\mathrm{MoS}_{2}$-graphene stacking regions. Therefore, nonlinear optical imaging can visualize the interlayer coupling effect for many van der Waals heterostructures. 


\section{CONCLUSIONS}

Multimodal nonlinear optical responses and imaging (FWM, SFG, SHG) of $\mathrm{MoS}_{2}$ and $\mathrm{MoS}_{2}-$ graphene heterostructures was studied using nonlinear optical microscopy. Our results demonstrated that $\mathrm{MoS}_{2}$ atomic layers exhibited strong second-(SFG, SHG) and third-order (FWM) nonlinear optical characteristics. All three types of nonlinearities were sensitive to the number of layers, crystallinity, and interlayer coupling. The combined multimodal nonlinear optical imaging allowed for the monitoring of chemical reactions in $\mathrm{MoS}_{2}$ and quantitative evaluation of $\mathrm{MoS}_{2}$ quality. $\mathrm{MoS}_{2}$-graphene heterostructures showed a strong quenching of SHG-SFG intensities, indicating strong interlayer coupling between $\mathrm{MoS}_{2}$ and graphene. This fast, versatile, and noninvasive multimodal nonlinear optical imaging approach is applicable to a broad range of 2D TMDs and TMD-based heterostructures to elucidate their fundamental structural and interlayer coupling properties. In addition, nonlinear optical imaging of chemical dynamics in van der Waals layered structures is also particularly important in terms of their potential applications in electronics, optoelectronics, and energy harvesting.

\section{METHODS}

\section{Sample fabrication}

(1) Mechanical exfoliation $\mathbf{M o S}_{2}$. Exfoliated $\mathrm{MoS}_{2}$ flakes with different layer thicknesses were prepared by microcleavage of natural bulk crystals (Graphene Supermarket) using Scotch $^{\circledR}$ Transparent Tape, before being transferred onto a transparent substrate, such as fused silica and sapphire. Monolayer and few-layer flakes were initially identified from the optical contrast using an optical microscope.

(2) Growth of $\mathrm{MoS}_{2}$ thin films. $\mathrm{MoS}_{2}$ thin films with controlled quality and uniformity were grown by solid-state sulfurization (SSS) of molybdenum (Mo) thin films evaporated on 
transparent sapphire substrates. The schematic of the $\mathrm{MoS}_{2}$ thin film growth process is shown in the Supporting Information, Figure S10a. First, a thin Mo film was deposited by sputtering; the film's thickness was controlled by adjusting the sputtering rate and time. The Mo films were then sulfurized in an argon/sulfur (Ar/S) atmosphere at temperatures between 800 and $1100{ }^{\circ} \mathrm{C}$. A schematic of the sulfurization process is shown in the Supporting Information, Figure S10b. Sulfur powder and samples were placed at the edge and the center of a thermal furnace, respectively. During the sulfurization process, sulfur powder evaporated and was transported above the Mo thin films deposited. The gaseous S atoms chemically interacted with the Mo atoms deposited in the high temperature environment to form atomically thin $\mathrm{MoS}_{2}$ films.

(3) Fabrication of $\mathrm{MoS}_{2}$-graphene heterostructures. $\mathrm{MoS}_{2}$-graphene heterostructures were produced by a dry transfer technique. ${ }^{58}$ First, a piece of glass slide was covered with polymer gel film (Gel-Pak Company) of approximately $1 \times 1 \mathrm{~cm}^{2}$ in size. A monolayer $\mathrm{MoS}_{2}$ flake was then exfoliated onto the gel film surface. Second, few-layer graphene was transferred onto a fused silica substrate via standard mechanical exfoliation. Third, the $\mathrm{MoS}_{2}$ flake on a gelfilm-covered glass substrate was positioned upside down and aligned with the graphene flake under an optical microscope with a long-working-distance lens. The two samples were brought into contact. Once completely contacted, both substrates were then slowly separated. As a result, $\mathrm{MoS}_{2}$ was transferred onto the graphene surfaces, forming the $\mathrm{MoS}_{2}$-graphene heterostructures.

\section{Multiphoton nonlinear optical spectroscopic imaging system}

The nonlinear optical properties of the prepared $\mathrm{MoS}_{2}$ and $\mathrm{MoS}_{2}$-graphene heterostructure samples were investigated using a homemade multiphoton nonlinear optical microscopic system (see Supporting Information, Figure S1). The design of this system has been 
previously reported. ${ }^{59}$ It mainly consisted of three parts: a laser source, a scanning module, and a signal detection module. Based on this nonlinear optical imaging system, various nonlinear optical signals could be simultaneously detected by multichannel photomultiplier tube (PMT) detectors. A commercial Ti:Sapphire femtosecond (fs) laser (MaiTai DeepSee HP, SpectraPhysics) provided a laser beam with a central wavelength of $800 \mathrm{~nm}$ (tunable from 780 to $820 \mathrm{~nm}$ ), a pulse duration of $100 \mathrm{fs}$, and a repetition of $80 \mathrm{MHz}$. The Ti:Sapphire fs laser, in conjunction with a supercontinuum generator (SCG-800, Newport), provided two incident laser beams whose powers can be controlled independently. One laser beam (probe light) was formed using a $500 \mathrm{~mW}$ laser beam to generate the supercontinuum using the supercontinuum generator, and then the laser beam was filtered through a long-pass filter (10CGA-830, Newport). The other laser beam (pump light) was formed by introducing the $800 \mathrm{~nm}$ laser beam through an attenuator and a delay line. The spectra of the pump-probe laser beams are shown in Supporting Information, Figure S2. The pump-probe laser beams were then focused collinearly onto the sample surfaces using a water-immersion objective lens with a numerical aperture of 1.05 and a working distance of $2 \mathrm{~mm}$. The focal spot size was measured to be $\sim 300 \mathrm{~nm}$. The signals were collected in the forward direction, where nonlinear optical spectra were detected using a spectrometer; and the second-/third-order nonlinear optical signals were split into three paths using a long-pass dichroic mirror and then detected using sensitive PMTs. The signal acquisition of the three channels was simultaneous, which made the measurement conditions for all channels identical regardless of any perturbations.

\section{Other characterization methods}

Raman and photoluminescence (PL) spectroscopy. Raman and PL spectral analyses were performed at room temperature in a micro-Raman spectrometer (Renishaw InVia plus, Renishaw, Gloucetershire, UK). The excitation source was an $\mathrm{Ar}^{+}$laser with a wavelength of 
$514.5 \mathrm{~nm}$ and a power of about $3 \mathrm{~mW}$. The spot size focused onto the sample was about 1 $\mu \mathrm{m}^{2}$. Raman and PL spectra were collected through a $50 \times$ objective lens with an accumulation time of $10 \mathrm{~s}$ at each position.

Atomic force microscopy. An AFM (Agilent 5500, CA, U.S.A) operated in the tapping mode was used to study the morphology and phase and to determine the thickness of van der Waals layered structures.

\section{ACKNOWLEDGEMENTS}

This research work was financially supported by the National Science Foundation (CMMI 1265122) and Nebraska Center for Energy Sciences Research.

\section{ASSOCIATED CONTENT}

Supporting Information Available: The Supporting Information is available free of charge on the ACS Publications website at http://pubs.acs.org. 



Figure 1. Multimodal nonlinear optical spectral analyses of a few-layer MoS2. (a) The schematic illustration of interactions of pump-probe laser beams with a $\mathrm{MoS}_{2}$ atomic crystal structure, where the second-(SHG, SFG) and third-order (FWM) nonlinear optical signals were simultaneously detected. FWM: four-wave mixing; SHG: second-harmonic generation; SFG: sum-frequency generation. The inset in (a) shows an optical image and corresponding Raman spectrum of the few-layer $\mathrm{MoS}_{2}$ tested, indicating a trilayer sample. Scale bar: $10 \mu \mathrm{m}$. (b) Nonlinear optical spectra generated by pump-probe laser beams. (c-e) Normalized nonlinear optical spectra excited with pump and/or probe beams of different wavelengths. (c) SHG dependence of the pump wavelength. (d) SFG dependence on pump-probe beams $\left(\lambda_{\text {pump }}\right.$, 
$\left.\lambda_{\text {probe }}\right)$ : $(785 \mathrm{~nm}, 957 \mathrm{~nm}),(794,998 \mathrm{~nm}),(803,1022 \mathrm{~nm})$. (e) FWM dependence on pumpprobe beams $\left(\lambda_{\text {pump }}, \lambda_{\text {probe }}\right)$ : $(785,1017 \mathrm{~nm}),(794,1019 \mathrm{~nm}),(803,1029 \mathrm{~nm})$. 

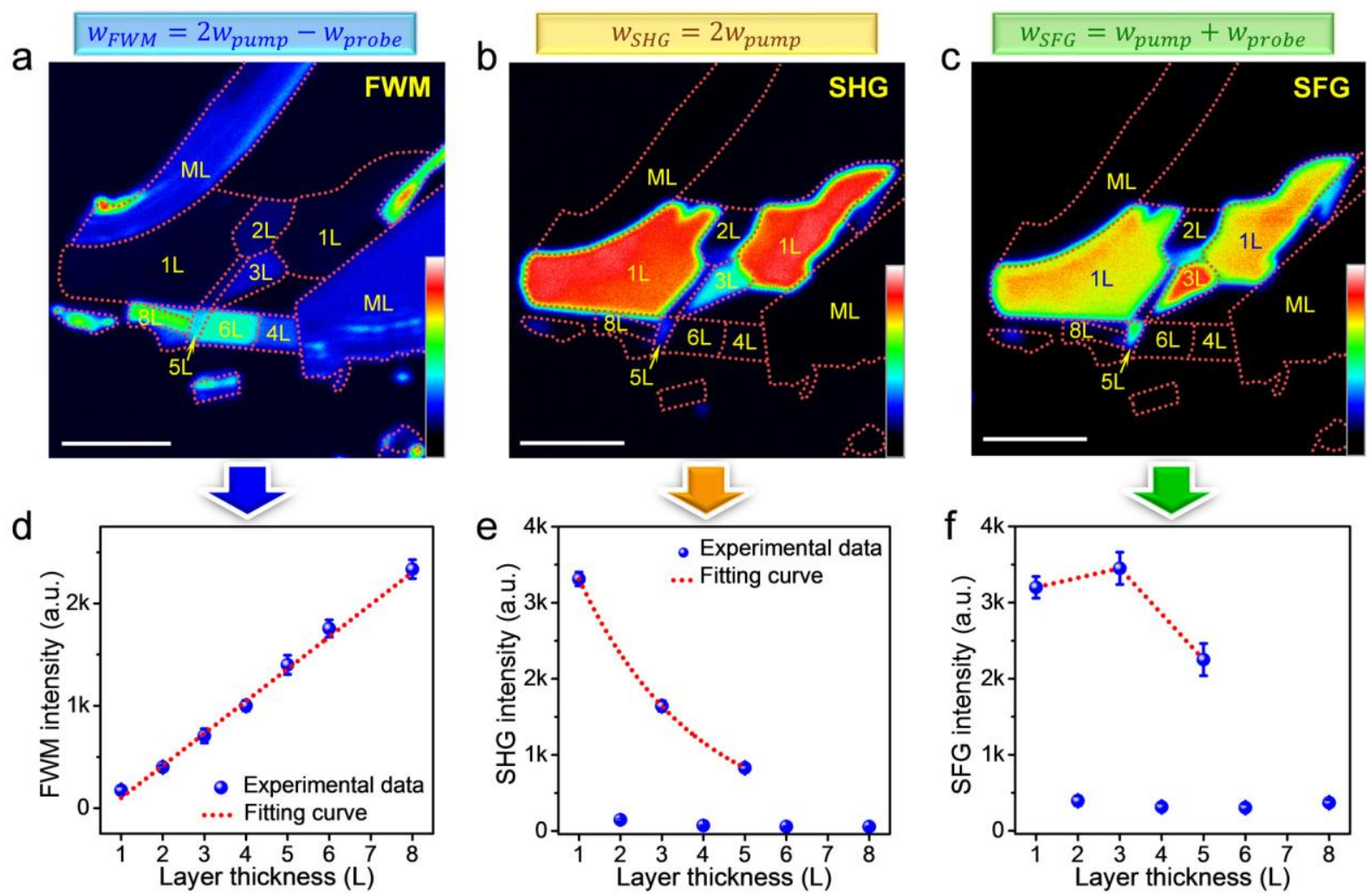

Figure 2. Multiphoton nonlinear optical images of a mechanically exfoliated few-layer

MoS 2 flakes with different thicknesses. (a) FWM, (b) SHG, and (c) SFG. Scale bar: $5 \mu \mathrm{m}$.

The dashed lines in (a-c) indicate the boundaries of the $\mathrm{MoS}_{2}$ flakes. The number of layers is also labeled. (d-f) Nonlinear optical signals of (d) FWM, (e) SHG and (f) SFG as functions of $\mathrm{MoS}_{2}$ layer thickness. 

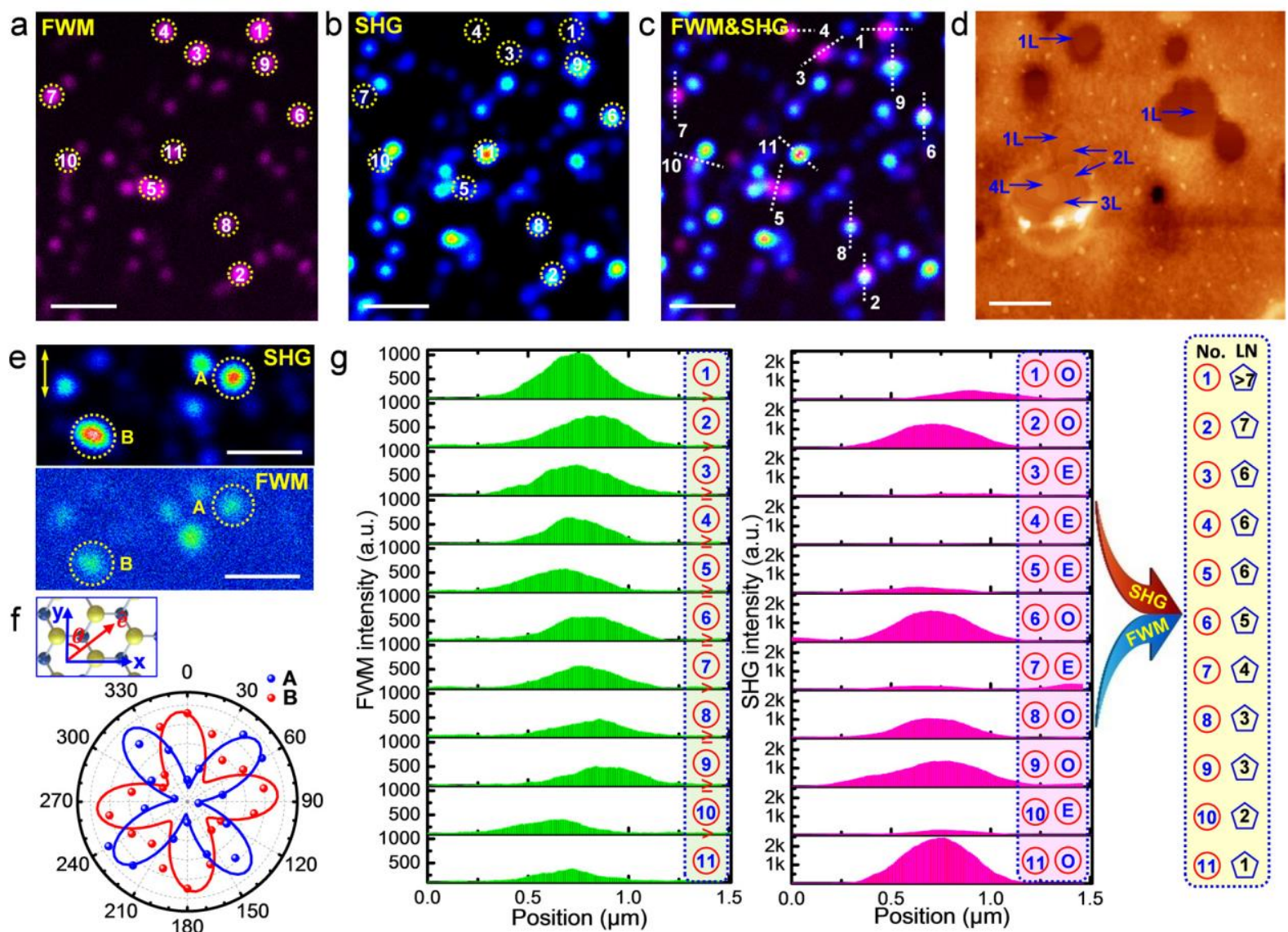

Figure 3. Comprehensive characterization of $\mathrm{MoS}_{2}$ thin films grown on sapphire substrate with superposed FWM-SHG analyses. (a) FWM, (b) SHG, (c) overlaid FWM$\mathrm{SHG}$, and (d) AFM images of a typical $\mathrm{MoS}_{2}$ thin film grown at $1100{ }^{\circ} \mathrm{C}$. (e) SHG and FWM images of two odd-layered $\mathrm{MoS}_{2}$ nanosheets in the same field. Scale bars: $2 \mu \mathrm{m}$ for $(\mathrm{a}, \mathrm{b}, \mathrm{c}, \mathrm{e})$ and $200 \mathrm{~nm}$ for (d). (f) Polar plot of the parallel polarization SHG intensity, at the locations indicated in $\mathrm{A}$ and $\mathrm{B}$ of (e), as functions of the incident pump polarization angle. $(\mathrm{g})$ Estimation of odd-even layers and layer numbers at different positions through superposed FWM-SHG analysis. 

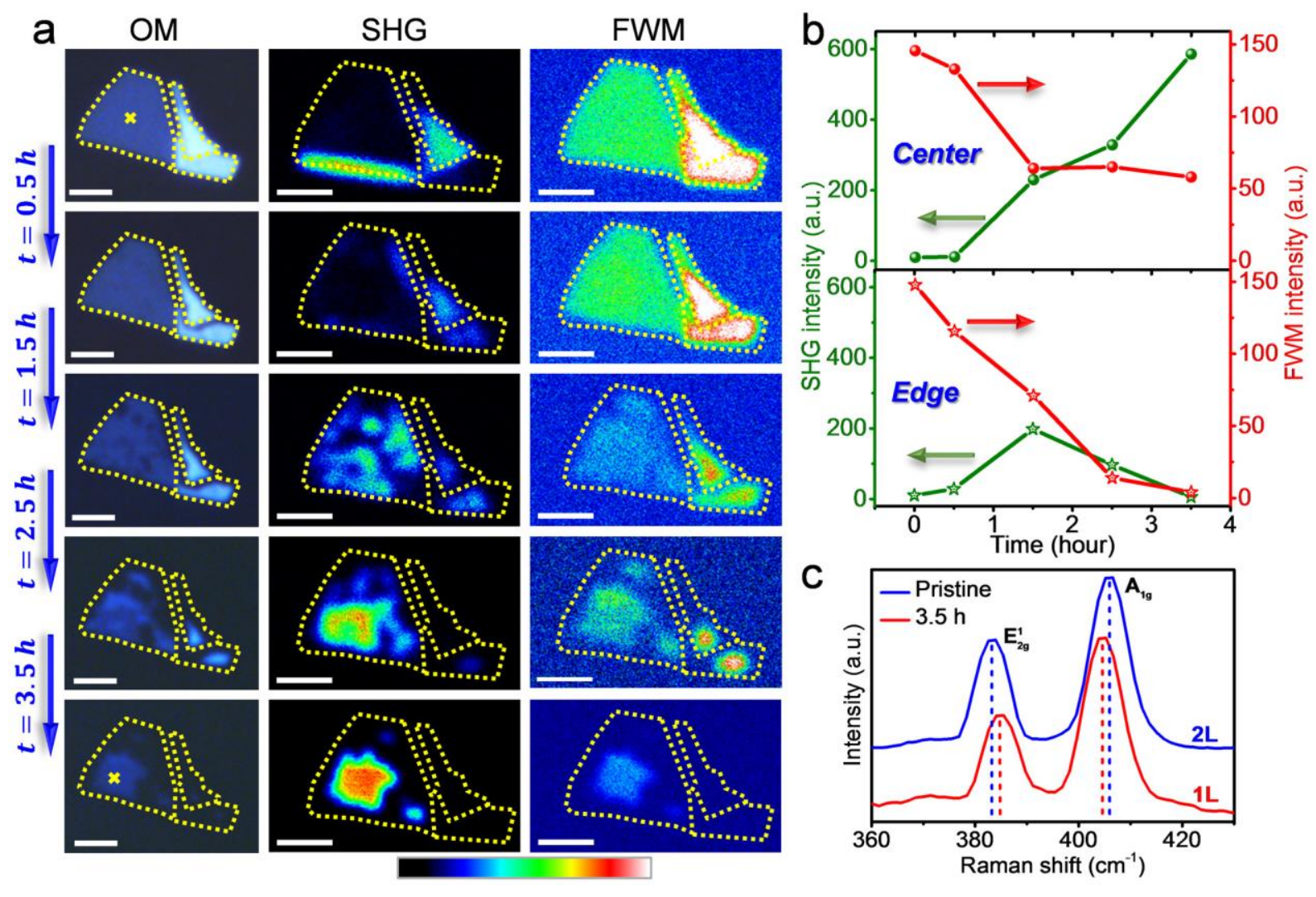

Figure 4. Thermal oxidation of a $\mathrm{MoS}_{2}$ flake after different heating times. (a, first column) Optical micrograph, (a, second column) SHG, and (a, third column) FWM images acquired in between several heating/cooling steps. The data are displayed in rows as a function of the heating time. Scale bar: $2 \mu \mathrm{m}$. (b) Changes in the SHG and FWM intensities at the center/edge areas with an increase in the heating time. (c) Raman spectra taken from both points as marked in (a). 



Figure 5. Nonlinear optical characterization of $\mathrm{MoS}_{2}$-graphene heterostructures. (a)

Optical micrograph, (b) AFM morphology, (c) SHG, (d) SFG, and (e) FWM images. Scale bar: $5 \mu \mathrm{m}$. The inset in (b) shows the cross-sectional height along the dashed line. (f-h) Nonlinear optical signals of (f) SHG, (g) SFG, and (h) FWM for the $\mathrm{MoS}_{2}-$ graphene heterostructure in the different regions. (i) Polar plots of the parallel polarization SHG intensity from the monolayer $\mathrm{MoS}_{2}$ and $1 \mathrm{Mo} / 2 \mathrm{G}$ stacking region as functions of the incident pump polarization angle. (j) Current versus drain bias curve for $\mathrm{MoS}_{2}$-graphene heterostructure $\left(V_{G}=0 \mathrm{~V}\right)$. The inset in $(j)$ shows the schematic of the $\mathrm{MoS}_{2}$-graphene heterostructure device for electric measurement and photocurrent scanning. (k) Photocurrent line scanning result $\left(\mathrm{V}_{\mathrm{SD}}=0.2 \mathrm{~V}\right)$ measured by scanning the laser beam from $\mathrm{MoS}_{2}$ to the stacking region and then to graphene of the heterostructure device. 


\section{REFERENCES}

1. Pu, J.; Yomogida, Y.; Liu, K.-K.; Li, L.-J.; Iwasa, Y.; Takenobu, T. Highly Flexible $\mathrm{MoS}_{2}$ Thin-Film Transistors with Ion Gel Dielectrics. Nano lett. 2012, 12, 4013-4017.

2. Qiu, H.; Pan, L.; Yao, Z.; Li, J.; Shi, Y.; Wang, X. Electrical Characterization of BackGated Bi-Layer $\mathrm{MoS}_{2}$ Field-Effect Transistors and the Effect of Ambient on Their Performances. Appl. Phys. Lett. 2012, 100, 123104.

3. Das, S.; Chen, H.-Y.; Penumatcha, A. V.; Appenzeller, J. High Performance Multilayer $\mathrm{MoS}_{2}$ Transistors with Scandium Contacts. Nano Lett. 2013, 13, 100-105.

4. Movva, H. C. P.; Rai, A.; Kang, S.; Kim, K.; Fallahazad, B.; Taniguchi, T.; Watanabe, K.; Tutuc, E.; Banerjee, S. K. High-Mobility Holes in Dual-Gated $\mathrm{WSe}_{2}$ Field-Effect Transistors. ACS Nano 2015, 9, 10402-10410.

5. Seyler, K. L.; Schaibley, J. R.; Gong, P.; Rivera, P.; Jones, A. M.; Wu, S.; Yan, J.; Mandrus, D. G.; Yao, W.; Xu, X. Electrical Control of Second-Harmonic Generation in a $\mathrm{WSe}_{2}$ Monolayer Transistor. Nat. Nanotechnol. 2015, 10, 407-411.

6. Radisavljevic, B.; Whitwick, M. B.; Kis, A. Integrated Circuits and Logic Operations Based on Single-Layer MoS2. ACS Nano 2011, 5, 9934-9938.

7. Wang, H.; Yu, L.; Lee, Y.-H.; Shi, Y.; Hsu, A.; Chin, M. L.; Li, L.-J.; Dubey, M.; Kong, J.; Palacios, T. Integrated Circuits Based on Bilayer $\mathrm{MoS}_{2}$ Transistors. Nano Lett. 2012, $12,4674-4680$.

8. Bernardi, M.; Palummo, M.; Grossman, J. C. Extraordinary Sunlight Absorption and One Nanometer Thick Photovoltaics Using Two-Dimensional Monolayer Materials. Nano Lett. 2013, 13, 3664-3670.

9. Radisavljevic, B.; Radenovic, A.; Brivio, J.; Giacometti, V.; Kis, A. Single-Layer MoS 2 Transistors. Nat. Nanotechnol. 2011, 6, 147-150. 
10. Yin, Z.; Li, H.; Li, H.; Jiang, L.; Shi, Y.; Sun, Y.; Lu, G.; Zhang, Q.; Chen, X.; Zhang, H. Single-Layer $\mathrm{MoS}_{2}$ Phototransistors. ACS Nano 2012, 6, 74-80.

11. Lopez-Sanchez, O.; Lembke, D.; Kayci, M.; Radenovic, A.; Kis, A. Ultrasensitive Photodetectors Based on Monolayer MoS 2. Nat. Nanotechnol. 2013, 8, 497-501.

12. Yun, J.-M.; Noh, Y.-J.; Yeo, J.-S.; Go, Y.-J.; Na, S.-I.; Jeong, H.-G.; Kim, J.; Lee, S.; Kim, S.-S.; Koo, H. Y.; Kim, T.-W.; Kim, D.-Y. Efficient Work-Function Engineering of Solution-Processed $\mathrm{MoS}_{2}$ Thin-Films for NovelHhole and Electron Transport Layers Leading to High-Performance Polymer Solar Cells. J. Mater. Chem. C 2013, 1, 37773783.

13. Laursen, A. B.; Kegnaes, S.; Dahl, S.; Chorkendorff, I. Molybdenum Sulfides-Efficient and Viable Materials for Electro - and Photoelectrocatalytic Hydrogen Evolution. Energy Environ. Sci. 2012, 5, 5577-5591.

14. Tan, Y.; Liu, P.; Chen, L.; Cong, W.; Ito, Y.; Han, J.; Guo, X.; Tang, Z.; Fujita, T.; Hirata, A.; Chen, M. W. Monolayer MoS 2 Films Supported by 3D Nanoporous Metals for High-Efficiency Electrocatalytic Hydrogen Production. Adv. Mater. 2014, 26, 8023-8028. 15. Liu, H.; Su, D.; Zhou, R.; Sun, B.; Wang, G.; Qiao, S. Z. Highly Ordered Mesoporous $\mathrm{MoS}_{2}$ with Expanded Spacing of the (002) Crystal Plane for Ultrafast Lithium Ion Storage. Adv. Energy Mater. 2012, 2, 970-975.

16. Anasori, B.; Xie, Y.; Beidaghi, M.; Lu, J.; Hosler, B. C.; Hultman, L.; Kent, P. R. C.; Gogotsi, Y.; Barsoum, M. W. Two-Dimensional, Ordered, Double Transition Metals Carbides (MXenes). ACS Nano 2015, 9, 9507-9516.

17. Yue, Q.; Shao, Z.; Chang, S.; Li, J. Adsorption of Gas Molecules on Monolayer $\mathrm{MoS}_{2}$ and Effect of Applied Electric Field. Nanoscale Res. lett. 2013, 8, 1-7. 
18. He, Q.; Zeng, Z.; Yin, Z.; Li, H.; Wu, S.; Huang, X.; Zhang, H. Fabrication of Flexible $\mathrm{MoS}_{2}$ Thin-Film Transistor Arrays for Practical Gas-Sensing Applications. Small 2012, 8, 2994-2999.

19. Perkins, F. K.; Friedman, A. L.; Cobas, E.; Campbell, P. M.; Jernigan, G. G.; Jonker, B. T. Chemical Vapor Sensing with Monolayer MoS 2 . Nano Lett. 2013, 13, 668-673.

20. Zeng, H.; Dai, J.; Yao, W.; Xiao, D.; Cui, X. Valley Polarization in $\mathrm{MoS}_{2}$ Monolayers by Optical Pumping. Nat. Nanotechnol. 2012, 7, 490-493.

21. Xu, X.; Yao, W.; Xiao, D.; Heinz, T. F. Spin and Pseudospins in Layered Transition Metal Dichalcogenides. Nat. Phys. 2014, 10, 343-350.

22. Xiao, D.; Liu, G.-B.; Feng, W.; Xu, X.; Yao, W. Coupled Spin and Valley Physics in Monolayers of $\mathrm{MoS}_{2}$ and Other Group-VI Dichalcogenides. Phys. Rev. Lett. 2012, 108, 196802.

23. Liu, J.; Zeng, Z.; Cao, X.; Lu, G.; Wang, L.-H.; Fan, Q.-L.; Huang, W.; Zhang, H. Preparation of $\mathrm{MoS}_{2}$-Polyvinylpyrrolidone Nanocomposites for Flexible Nonvolatile Rewritable Memory Devices with Reduced Graphene Oxide Electrodes. Small 2012, 8, $3517-3522$.

24. Bertolazzi, S.; Krasnozhon, D.; Kis, A. Nonvolatile Memory Cells Based on $\mathrm{MoS}_{2} /$ Graphene Heterostructures. ACS Nano 2013, 7, 3246-3252.

25. Lei, S.; Wen, F.; Li, B.; Wang, Q.; Huang, Y.; Gong, Y.; He, Y.; Dong, P.; Bellah, J.; George, A.; Ge, L.; Lou, J.; Halas, N. J.; Vajtai, R.; Ajayan, P. M. Optoelectronic Memory Using Two-Dimensional Materials. Nano Lett. 2015, 15, 259-265.

26. Hamm, J. M.; Hess, O. Two Two-Dimensional Materials Are Better than One. Science 2013, 340, 1298-1299. 
27. Shih, C.-J.; Wang, Q. H.; Son, Y.; Jin, Z.; Blankschtein, D.; Strano, M. S. Tuning On-Off Current Ratio and Field-Effect Mobility in a $\mathrm{MoS}_{2}-$ Graphene Heterostructure via Schottky Barrier Modulation. ACS Nano 2014, 8, 5790-5798.

28. Cui, X.; Lee, G.-H.; Kim, Y. D.; Arefe, G.; Huang, P. Y.; Lee, C.-H.; Chenet, D. A.; Zhang, X.; Wang, L.; Ye, F.; Pizzocchero, F.; Jessen, B. S.; Watanabe, K.; Taniguchi, T.; Muller, D. A.; Low, T.; Kim, P.; Hone, J. Multi-Terminal Transport Measurements of $\mathrm{MoS}_{2}$ Using a van der Waals Heterostructure Device Platform. Nat. Nanotechnol. 2015, $10,534-540$.

29. Liu, Y.; Wu, H.; Cheng, H.-C.; Yang, S.; Zhu, E.; He, Q.; Ding, M.; Li, D.; Guo, J.; Weiss, N. O.; Huang, Y.; Duan, X. Toward Barrier Free Contact to Molybdenum Disulfide Using Graphene Electrodes. Nano Lett. 2015, 15, 3030-3034.

30. Lee, C.; Yan, H.; Brus, L. E.; Heinz, T. F.; Hone, J.; Ryu, S. Anomalous Lattice Vibrations of Single- and Few-Layer MoS 2 . ACS Nano 2010, 4, 2695-2700.

31. Li, H.; Zhang, Q.; Yap, C. C. R.; Tay, B. K.; Edwin, T. H. T.; Olivier, A.; Baillargeat, D. From Bulk to Monolayer $\mathrm{MoS}_{2}$ : Evolution of Raman Scattering. Adv. Funct. Mater. 2012, $22,1385-1390$.

32. Zhao, Y.; Luo, X.; Li, H.; Zhang, J.; Araujo, P. T.; Gan, C. K.; Wu, J.; Zhang, H.; Quek, S. Y.; Dresselhaus, M. S. Interlayer Breathing and Shear Modes in Few-Trilayer $\mathrm{MoS}_{2}$ and WSe 2 . Nano lett. 2013, 13, 1007-1015.

33. Mak, K. F.; Lee, C.; Hone, J.; Shan, J.; Heinz, T. F. Atomically Thin $\mathrm{MoS}_{2}$ : a New Direct-Gap Semiconductor. Phys. Rev. Lett. 2010, 105, 136805.

34. Splendiani, A.; Sun, L.; Zhang, Y.; Li, T.; Kim, J.; Chim, C.-Y.; Galli, G.; Wang, F. Emerging Photoluminescence in Monolayer MoS 2 . Nano Lett. 2010, 10, 1271-1275. 
35. Zeng, H.; Liu, G.-B.; Dai, J.; Yan, Y.; Zhu, B.; He, R.; Xie, L.; Xu, S.; Chen, X.; Yao, W. Optical Signature of Symmetry Variations and Spin-Valley Coupling in Atomically Thin Tungsten Dichalcogenides. Sci. Rep. 2013, 3.

36. Kumar, N.; Najmaei, S.; Cui, Q.; Ceballos, F.; Ajayan, P. M.; Lou, J.; Zhao, H. Second Harmonic Microscopy of Monolayer $\mathrm{MoS}_{2}$. Phys. Rev. B 2013, 87, 161403.

37. Li, Y.; Rao, Y.; Mak, K. F.; You, Y.; Wang, S.; Dean, C. R.; Heinz, T. F. Probing Symmetry Properties of Few-Layer $\mathrm{MoS}_{2}$ and h-BN by Optical Second-Harmonic Generation. Nano Lett. 2013, 13, 3329-3333.

38. Yin, X.; Ye, Z.; Chenet, D. A.; Ye, Y.; O’Brien, K.; Hone, J. C.; Zhang, X. Edge Nonlinear Optics on a $\mathrm{MoS}_{2}$ Atomic Monolayer. Science 2014, 344, 488-490.

39. David, S. N.; Zhai, Y.; van der Zande, A. M.; apos; Brien, K.; Huang, P. Y.; Chenet, D. A.; Hone, J. C.; Zhang, X.; Yin, X. Rapid, All-Optical Crystal Orientation Imaging of Two-Dimensional Transition Metal Dichalcogenide Monolayers. Appl. Phys. Lett. 2015, 107, 111902.

40. Hendry, E.; Hale, P. J.; Moger, J.; Savchenko, A.; Mikhailov, S. Coherent Nonlinear Optical Response of Graphene. Phys. Rev. Lett. 2010, 105, 097401.

41. Malard, L. M.; Alencar, T. V.; Barboza, A. P. M.; Mak, K. F.; de Paula, A. M. Observation of Intense Second Harmonic Generation From $\mathrm{MoS}_{2}$ Atomic Crystals. Phys. Rev. B 2013, 87, 201401.

42. Chiappe, D.; Asselberghs, I.; Sutar, S.; Iacovo, S.; Afanas'ev, V.; Stesmans, A.; Balaji, Y.; Peters, L.; Heyne, M.; Mannarino, M.; Vandervorst, W.; Sayan, S.; Huyghebaert, C.; Caymax, M.; Heyns, M.; De Gendt, S.; Radu, I.; Thean, A. Controlled Sulfurization Process for the Synthesis of Large Area $\mathrm{MoS}_{2}$ Films and $\mathrm{MoS}_{2} / \mathrm{WS}_{2}$ Heterostructures. Adv. Mater. Interfaces 2015, 1500635. 
43. Tarasov, A.; Campbell, P. M.; Tsai, M.-Y.; Hesabi, Z. R.; Feirer, J.; Graham, S.; Ready, W. J.; Vogel, E. M. Highly Uniform Trilayer Molybdenum Disulfide for Wafer-Scale Device Fabrication. Adv. Funct. Mater. 2014, 24, 6389-6400.

44. Bessonov, A. A.; Kirikova, M. N.; Petukhov, D. I.; Allen, M.; Ryhänen, T.; Bailey, M. J. A. Layered Memristive and Memcapacitive Switches for Printable Electronics. Nat. Mater. 2015, 14, 199-204.

45. Castellanos-Gomez, A.; Barkelid, M.; Goossens, A. M.; Calado, V. E.; van der Zant, H. S. J.; Steele, G. A. Laser-Thinning of $\mathrm{MoS}_{2}$ : On Demand Generation of a Single-Layer Semiconductor. Nano Lett. 2012, 12, 3187-3192.

46. Yamamoto, M.; Dutta, S.; Aikawa, S.; Nakaharai, S.; Wakabayashi, K.; Fuhrer, M. S.; Ueno, K.; Tsukagoshi, K. Self-Limiting Layer-by-Layer Oxidation of Atomically Thin WSe 2 . Nano Lett. 2015, 15, 2067-2073.

47. Liu, Y.; Tan, C.; Chou, H.; Nayak, A.; Wu, D.; Ghosh, R.; Chang, H.-Y.; Hao, Y.; Wang, X.; Kim, J.-S.; Piner, R.; Ruoff, R. S.; Akinwande, D.; Lai, K. Thermal Oxidation of WSe 2 Nanosheets Adhered on $\mathrm{SiO}_{2} / \mathrm{Si}$ Substrates. Nano Lett. 2015, 15, 4979-4984.

48. Liu, W.-T.; Wu, S. W.; Schuck, P. J.; Salmeron, M.; Shen, Y. R.; Wang, F. Nonlinear Broadband Photoluminescence of Graphene Induced by Femtosecond Laser Irradiation. Phys. Rev. B 2010, 82, 081408.

49. Wang, R.; Chien, H.-C.; Kumar, J.; Kumar, N.; Chiu, H.-Y.; Zhao, H. Third-Harmonic Generation in Ultrathin Films of $\mathrm{MoS}_{2}$. ACS Appl. Mater. Inter. 2014, 6, 314-318.

50. Hsu, W.-T.; Zhao, Z.-A.; Li, L.-J.; Chen, C.-H.; Chiu, M.-H.; Chang, P.-S.; Chou, Y.-C.; Chang, W.-H. Second Harmonic Generation From Artificially Stacked Transition Metal Dichalcogenide Twisted Bilayers. ACS Nano 2014, 8, 2951-2958.

51. Grüning, M.; Attaccalite, C. Second Harmonic Generation in h-BN and $\mathrm{MoS}_{2}$ Monolayers: Role of Electron-Hole Interaction. Phys. Rev. B 2014, 89, 081102. 
52. Buscema, M.; Steele, G. A.; van der Zant, H. S.; Castellanos-Gomez, A. The Effect of the Substrate on the Raman and Photoluminescence Emission of Single-Layer MoS 2 . Nano Res. 2014, 7, 561-571.

53. Rathi, S.; Lee, I.; Lim, D.; Wang, J.; Ochiai, Y.; Aoki, N.; Watanabe, K.; Taniguchi, T.; Lee, G.-H.; Yu, Y.-J.; Kim, P.; Kim, G.-H. Tunable Electrical and Optical Characteristics in Monolayer Graphene and Few-Layer $\mathrm{MoS}_{2}$ Heterostructure Devices. Nano Lett. 2015, $15,5017-5024$.

54. Yu, W. J.; Liu, Y.; Zhou, H.; Yin, A.; Li, Z.; Huang, Y.; Duan, X. Highly Efficient GateTunable Photocurrent Generation in Vertical Heterostructures of Layered Materials. Nat. Nanotechnol. 2013, 8, 952-958.

55. Song, J.; Lu, H.; Gruverman, A.; Ducharme, S. Polarization Imaging in Ferroelectric Polymer Thin Film Capacitors by Pyroelectric Scanning Microscopy. Appl. Phys. Lett. 2014, 104, 192901.

56. Chiu, M.-H.; Li, M.-Y.; Zhang, W.; Hsu, W.-T.; Chang, W.-H.; Terrones, M.; Terrones, H.; Li, L.-J. Spectroscopic Signatures for Interlayer Coupling in $\mathrm{MoS}_{2}-\mathrm{WSe}_{2}$ van der Waals Stacking. ACS Nano 2014, 8, 9649-9656.

57. Fang, H.; Battaglia, C.; Carraro, C.; Nemsak, S.; Ozdol, B.; Kang, J. S.; Bechtel, H. A.; Desai, S. B.; Kronast, F.; Unal, A. A. Strong Interlayer Coupling in van der Waals Heterostructures Built From Single-Layer Chalcogenides. P. Natl. Acad. Sci. 2014, 111, 6198-6202.

58. Castellanos-Gomez, A.; Buscema, M.; Molenaar, R.; Singh, V.; Janssen, L.; van der Zant, H. S.; Steele, G. A. Deterministic Transfer of Two-Dimensional Materials by All-Dry Viscoelastic Stamping. 2D Mater. 2014, 1, 011002. 
59. Li, D. W.; Zhou, Y. S.; Huang, X.; Jiang, L.; Silvain, J. F.; Lu, Y. F. In Situ Imaging and Control of Layer-by-Layer Femtosecond Laser Thinning of Graphene. Nanoscale 2015, 7, 3651-3659.

\section{Table of Contents Graphic}

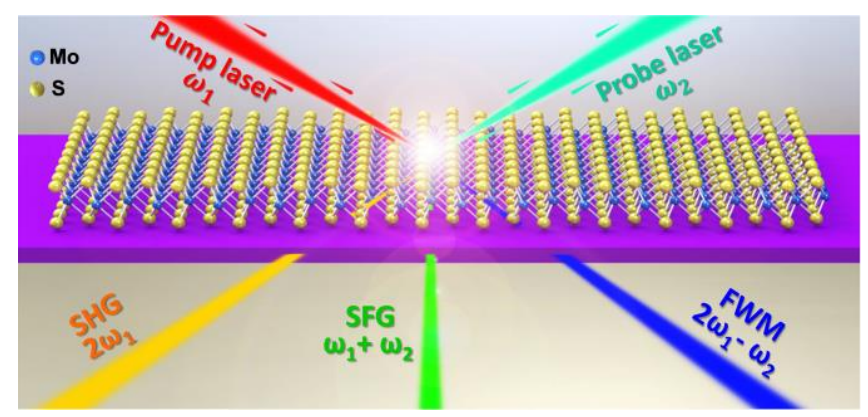

